\title{
Estudo correlacional entre parâmetros de avaliação da marcha e equilíbrio em idosas institucionalizadas
}

\section{Correlation study between gait and balance assessment parameters in institutionalized elderly women}

\author{
Paulo Adão de Medeiros'; Camila Heinzz; Lidiane Isabel Filippin ${ }^{3}$; Alecsandra Pinheiro \\ Vendrusculo4; Janeisa Franck Virtuoso5; Inês Amanda Streit6; Giovana Zarpellon Mazo7 \\ 'Fisioterapeuta, Especialista em Atividade Física, Desempenho Motor e Saúde - UFSM. Santa Maria, RS - Brasil. \\ ${ }^{2}$ Acadêmica do Curso de Fisioterapia - UNIFRA. Santa Maria, RS - Brasil. \\ ${ }^{3}$ Fisioterapeuta, Doutora em Ciências Médicas e Docente do Curso de Fisioterapia - UNIFRA. Santa Maria, RS - Brasil. \\ ${ }^{4}$ Fisioterapeuta, Mestre em Ciências do Movimento Humano e Docente do Curso de Fisioterapia - UNIFRA. Santa Maria, RS - Brasil. \\ ${ }^{5}$ Fisioterapeuta, Doutoranda em Ciências do Movimento Humano - UDESC. Florianópolis, SC - Brasil. \\ ${ }^{6}$ Licenciada em Educação Física, Mestranda em Ciências do Movimento Humano - UDESC. Florianópolis, SC - Brasil. \\ ${ }^{7}$ Doutora em Educação Física, Professora - UDESC, Centro de Ciências da Saúde e do Esporte. Florianópolis, SC - Brasil. \\ Endereço para Correspondência \\ Paulo Adão de Medeiros \\ Rua Daudt, 197 \\ 97010-150 - Santa Maria, RS - Brasil \\ paulofisiosm@yahoo.com.br
}

\begin{abstract}
Resumo
Objetivo: Correlacionar parâmetros de avaliação da marcha e equilíbrio em idosas institucionalizadas. Métodos: Foram avaliadas 36 idosas ( $71,92 \pm 8,48$ anos) e aplicados o teste de um minuto de marcha para avaliação da cadência da marcha, o teste de velocidade da marcha e a Avaliação da Marcha e Equilíbrio Orientada pelo Desempenho (POMA). Utilizou-se estatística descritiva e inferencial por meio do teste de correlação de Pearson (r). Adotou-se um nível de significância de 5\%. Resultados: Observou-se correlação positiva e significativa entre cadência e velocidade da marcha $(r=0,649 ; p<0,001)$, e entre o equilíbrio com a velocidade $(\mathrm{r}=0,641 ; \mathrm{p}<0,001)$ e com a cadência da marcha $(\mathrm{r}=0,398 ; \mathrm{p}=0,016)$. Conclusão: Existe correlação entre os parâmetros analisados, sendo maior entre o desempenho na marcha e equilíbrio com a velocidade da marcha. Recomendam-se novas investigações que venham contemplar relações entre componentes da aptidão muscular e os parâmetros de avaliação da marcha e equilíbrio.
\end{abstract}

Descritores: Idosos; Institucionalização; Equilíbrio postural; Marcha.

\begin{abstract}
Objective: To correlate gait assessment parameters in institutionalized elderly women. Methods: A total of 36 elderly women (71.92 \pm 8.48 years) were evaluated through the application of the one-minute walk test for the assessment of gait cadence, the gait speed test and the Performance-Oriented Evaluation of Gait and Balance (POMA). Descriptive and inferential statistics were gathered with Pearson's correlation $(r)$ test. A significance level of 5\% was adopted. Results: There was a positive and significant correlation between cadence and gait velocity $(r=0.649, p<0.001)$, and between balance and speed $(r=0.641, p<0.001)$ and gait cadence $(r=0.398, p=0.016)$. Conclusion: There is a correlation between the analyzed parameters, being higher between gait speed and performance in gait and balance. Further investigations bearing on the relation between muscular fitness components and gait and balance assessment parameters are recommended.
\end{abstract} Key words: Elderly; Gait; Institutionalization; Postural balance. 


\section{Introdução}

O envelhecimento populacional ocorre em meio a grandes mudanças na sociedade e na reconfiguração dos arranjos familiares. Assim, o Estado e o mercado privado passam a dividir com a família as responsabilidades no cuidado com a população idosa, tornando as instituições de longa permanência para idosos (ILPI) uma das alternativas de cuidados não familiares existentes ${ }^{1}$.

Junto ao processo de envelhecer, geralmente ocorre um declínio da aptidão física dos indivíduos, porém com o sedentarismo essa condição pode agravar-se levando até mesmo a dependência de cuidados ${ }^{2}$. Observa-se que a institucionalização por si só representa um fator de risco, já que os idosos institucionalizados necessitam de atenção, suporte e serviços especializados, pois a grande maioria é fragilizada e apresenta morbidades físicas ou mentais. Pelo seu isolamento social, inatividade física e processos psicológicos, subentende-se que quanto maior o tempo de institucionalização, maior a debilidade do idoso ${ }^{3}$.

Dentre as perdas funcionais, as vias responsáveis pelo equilíbrio corporal são significativamente acometidas, provocando alterações no sistema proprioceptivo, visual ou vestibular, dificultando e até impossibilitando a locomoção independente ${ }^{4}$, o que compromete a realização das atividades da vida diária ${ }^{5}$. Nos idosos, a marcha tem sido investigada, em razão da importância que a manutenção da mobilidade representa para esse grupo de indivíduos, em termos de autonomia e qualidade de vida ${ }^{6,7}$. Do mesmo modo, pode-se relacionar o estudo da marcha à prevenção de quedas, já que o prejuízo da capacidade funcional parece ter um papel preponderante na sua interação multicausal ${ }^{5}$.

$\mathrm{Na}$ avaliação da marcha, são necessárias medidas claras e objetivas ao invés de julgamentos subjetivos, pois uma análise apurada quantifica o andar e contribui para a tomada de decisões terapêuticas ${ }^{8}$. Para isso, existem vários testes que buscam avaliar os componentes da marcha, como, por exemplo, a velocidade que é considerada o parâmetro isolado que melhor representa a performance da marcha9. A amplitude e cadência do passo também são características essenciais da marcha humana e podem representar uma forma rápida e simples de verificar a fragilidade do idoso nessa importante atividade do cotidiano ${ }^{10}$.

Ainda segundo Matsudo ${ }^{11}$, alterações na mobilidade são um prognóstico negativo para a manutenção da capacidade funcional em idosos, pois indivíduos com a mobilidade prejudicada possuem maior risco de mortalidade e morbidades do que aqueles que mantêm a mobilidade preservada. Portanto, considerando que, à medida que se envelhece, o corpo sofre mudanças que influenciarão em todos os componentes da marcha e no equilíbrio e sendo poucos os estudos que avaliam as condições físicas dos idosos institucionalizados ${ }^{12,13}$, nesta pesquisa, buscaram-se correlacionar parâmetros de avaliação da marcha e equilíbrio em um grupo de idosas institucionalizadas na cidade de Santa Maria - RS.

\section{Materiais e método}

Este estudo transversal foi realizado em uma Instituição de Longa Permanência para Idosos (ILPI) que abriga mulheres carentes no município de Santa Maria, região central do Rio Grande do Sul. A pesquisa foi desenvolvida entre abril e julho de 2011, período em que residiam no local 196 mulheres; e, para composição da amostra, foram selecionadas aquelas com idade superior a 60 anos que apresentavam deambulação independente, com ou sem o auxílio de dispositivo de marcha e não tinham deficiências físicas limitantes. As idosas também foram avaliadas por meio do MiniExame do Estado Mental (MEEM) para identificar aquelas com boas condições cognitivas para entender os comandos solicitados, segundo o ponto de corte adotado por Bertolucci et al. ${ }^{14}$. Dessa forma, 39 idosas foram seleciona- 
das, exceto três que se recusaram a participar do estudo, totalizando uma amostra de 36 idosas, cuja média de idade foi 71,92 anos ( $\mathrm{DP}=$ $\pm 8,48$ ), variando de 60 a 94,9 anos.

Foram utilizados três instrumentos para avaliação da marcha e do equilíbrio das participantes. Para avaliação da cadência da marcha, utilizou-se a prova de um minuto de marcha adaptado de Viel ${ }^{15}$, que foi realizada sem interrupção, em uma pista de 18 metros de comprimento, sendo contabilizados apenas os passos nos dez metros centrais, no qual a velocidade permaneceu constante e o mais próxima da realidade no período de um minuto. Os quatro primeiros e últimos metros dessa pista foram utilizados como recuos para aceleração e desaceleração, sendo os passos e o tempo gasto nesse percurso desprezados.

Para avaliação da velocidade da marcha, realizou-se um teste adaptado de Abreu e Caldas ${ }^{16}$ em que as idosas foram orientadas a deambular em um ritmo habitual em uma segunda pista, ao longo de um percurso de 14 metros de comprimento, sendo os três primeiros e os três últimos metros desprezados para a aceleração e desaceleração, respectivamente. Portanto, foi medido o tempo gasto para completar os oito metros centrais; dessa forma, o resultado foi calculado pela média do tempo de três tentativas e, assim, expresso em metros por segundo $(\mathrm{m} / \mathrm{s})$.

O último teste aplicado foi a Avaliação da Marcha e Equilíbrio Orientada pelo Desempenho (ou POMA, do Inglês PerformanceOriented Mobility Assessment), desenvolvido por Tinetti ${ }^{17}$ que foi validado e adaptado para a população brasileira ${ }^{18}$ e busca avaliar o desempenho na marcha e equilíbrio de idosos e, portanto, pode ser usado para identificar risco de quedas. É composto por duas etapas: no primeiro momento foi avaliado o equilíbrio da idosa, em uma escala de 1 a 9 quesitos. Posteriormente, foi analisado o desempenho da marcha, em uma escala de continuidade da primeira de 10 a 16 quesitos.
Todos os testes foram aplicados pelo mesmo pesquisador, previamente treinado, no mesmo período do dia (turno da tarde), e antes do início da coleta de dados foram realizados cinco testes-piloto com idosas que não se enquadram na amostra, para adequação e familiarização com os instrumentos.

O tratamento estatístico dos dados se deu de maneira descritiva (média e desvio-padrão) e inferencial por meio do teste de correlação de Pearson (r). Adotou-se um nível de significância de $5 \%$.

Foram respeitadas as diretrizes e normas regulamentadoras para pesquisa com seres humanos, apontadas pela Resolução 196/96, do Conselho Nacional de Saúde ${ }^{19}$, sendo o estudo aprovado pelo Comitê de Ética em Pesquisa do Centro Universitário Franciscano - UNIFRA, sob o protocolo $\mathrm{n}^{\mathrm{o}} 436.2010 .2$.

\section{Resultados}

Foram avaliadas 36 idosas com média de idade de 71,92 anos $(\mathrm{DP}= \pm 8,48)$. O tempo médio de institucionalização foi $9,95(\mathrm{DP}= \pm 11,07)$ anos. Com relação aos parâmetros de marcha e equilíbrio, expostos na Tabela 1, nota-se que a velocidade média das idosas institucionalizadas foi 0,63 m/s, enquanto a cadência foi 89,39 passos por minuto. Quanto ao desempenho da marcha e do equilíbrio, a pontuação média das participantes foi 22,97 pontos.

Tabela l: Média e Intervalo de Confiança da velocidade, cadência e desempenho da marcha e equilíbrio de idosas institucionalizadas $(\mathrm{n}=36)$

\begin{tabular}{ccc}
\hline Variáveis do estudo & Média & IC (95\%) \\
\hline $\begin{array}{c}\text { Velocidade da marcha } \\
\text { (m/s) }\end{array}$ & 0,63 & $0,56-0,71$ \\
$\begin{array}{c}\text { Cadência da marcha } \\
\text { (passos/minuto) }\end{array}$ & 89,39 & $83,95-94,83$ \\
$\begin{array}{c}\text { Desempenho na marcha } \\
\text { e equilíbrio (pontuação) }\end{array}$ & 22,97 & $21,85-24,10$ \\
\hline
\end{tabular}

IC= Intervalo de Confiança; $\mathrm{m} / \mathrm{s}=$ metros por segundo. 
Na correlação entre as variáveis, observase no gráfico da Figura 1 a existência de correlação moderada, positiva e significativa entre cadência e velocidade da marcha $(r=0,649 ; \mathrm{p}<$ $0,001)$, de modo que, quanto maior a velocidade $(\mathrm{m} / \mathrm{s})$ também maior será o número de passos dados em um minuto. O coeficiente de determinação $\left(R_{2}\right)$ aponta que $42,1 \%$ da variação da velocidade da marcha ocorrem pela variação da cadência.

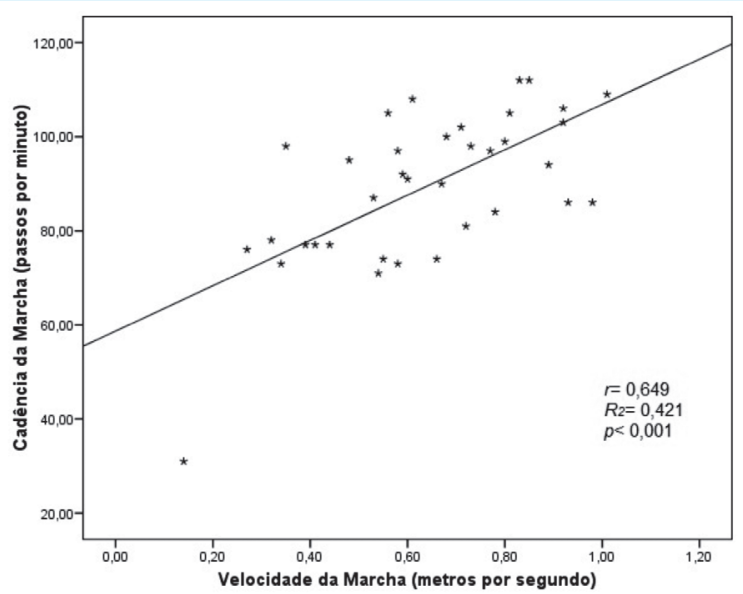

Figura 1: Correlação entre Cadência e Velocidade da Marcha $\mathrm{r}=$ estatística da correlação de Pearson; R2= coeficiente de determinação; $p=$ nível de significância.

Quanto ao desempenho da marcha e do equilíbrio, nota-se correlação positiva e significativa com a velocidade e a cadência da marcha (Figuras 2 e 3). A pontuação obtida nessa avaliação tende a aumentar quanto maior for a velocidade $(r=0,641 ; p<0,001)$ e a cadência da marcha $(r=0,398 ; p=0,016)$. Quanto ao coeficiente de determinação $\left(R_{2}\right)$, observou-se que $41 \%$ da variação do desempenho na marcha e equilíbrio ocorrem pela variação da velocidade da marcha, enquanto apenas $15,8 \%$ podem ser explicados pela cadência da marcha. Ainda pode-se observar que a correlação entre o desempenho da marcha e o equilíbrio com a velocidade da marcha (Figura 2) é considerada moderada, enquanto que com a cadência da marcha (Figura 3) trata-se de uma correlação fraca.

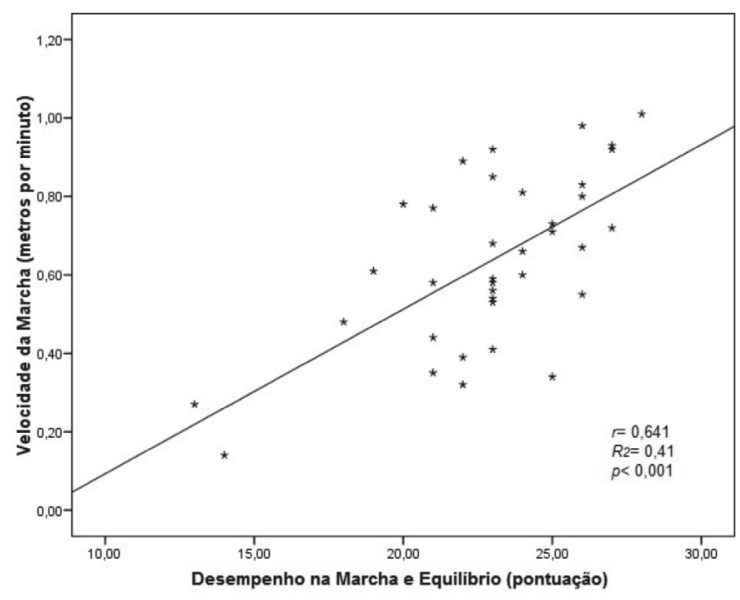

Figura 2: Correlação entre Velocidade da Morcha e Desempenho na Morcha e Equilíbrio $\mathrm{r}=$ estatística da correlação de Pearson; R2= coeficiente de determinação; $p=$ nível de significância.

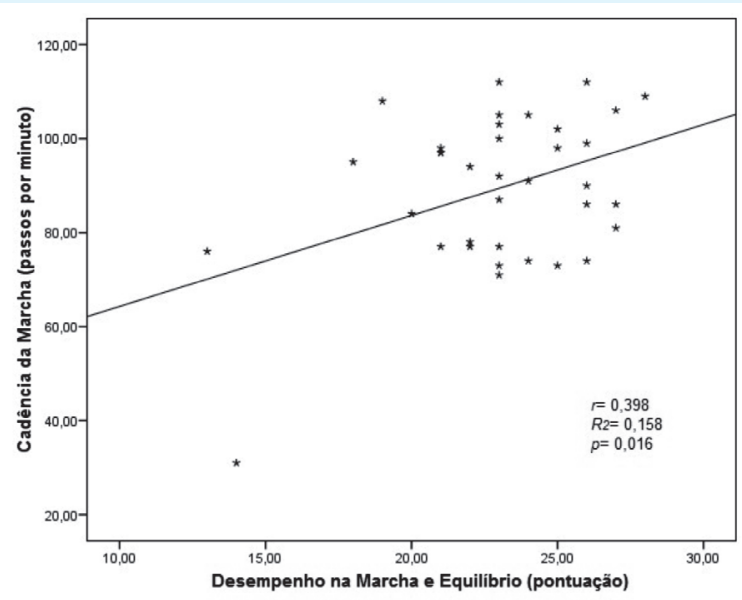

Figura 3: Correlação entre Cadência da Marcha e Desempenho na Marcha e Equilíbrio $r=$ estatística da correlação de Pearson; $R 2=$ coeficiente de determinação; $p=$ nível de significância.

\section{Discussão}

Neste estudo, teve-se como objetivo correlacionar parâmetros de avaliação da marcha e equilíbrio em idosas institucionalizadas. Por meio desta pesquisa foi possível observar uma significativa correlação entre o desempenho na marcha e equilíbrio, parâmetro utilizado para identificar potencial risco de quedas, e a velocidade da marcha. Esse resultado faz associação com o que foi observado em estudo que 
avaliou a velocidade de marcha de um grupo de idosos ${ }^{20}$, distinguindo-os em três níveis de velocidade (alta, média e baixa), e no qual se verificou que os idosos com menor velocidade de marcha foram os que apresentaram alta incidência de quedas.

Assim, a redução da velocidade de marcha em idosos sugere uma diminuição da capacidade de controlar o equilíbrio corporal ou, ainda, uma dificuldade na manutenção do equilíbrio durante a marcha, fazendo com que os idosos tenham maiores fases de apoio e menores fases de balanço, comparados com populações mais jovens e, por conseguinte, provocando uma diminuição da velocidade ${ }^{21}$.

No entanto, Abreu e Caldas ${ }^{16}$, ao estudar a velocidade da marcha, equilíbrio e idade entre idosas praticantes e não praticantes de exercícios não evidenciaram correlação entre a velocidade de marcha e o equilíbrio que pudesse apoiar a ideia de que os idosos estariam utilizando a redução da velocidade como uma estratégia compensatória para assegurar um equilíbrio adequado. Além disso, outros estudos indicam que a redução da velocidade da marcha entre os idosos esteja associada a mudanças estruturais do aparelho locomotor ligadas ao processo de envelhecimento, como a redução da força e elasticidade muscular, da mobilidade articular entre outras alterações biomecânicas ${ }^{22,23}$.

Nesse sentido, a correlação entre o desempenho na marcha e equilíbrio e a cadência da marcha também poderia ser explicada pela deterioração das condições físicas dos idosos, pois já foi evidenciado que a cadência da marcha na população idosa está associada aos componentes de aptidão muscular, mais especificamente, à flexibilidade, força e resistência ${ }^{10}$. Em outro estudo recente ${ }^{24}$ os autores sugerem que existe associação entre as variáveis da aptidão física muscular com a faixa etária, tendo essas forte influência sobre a marcha. Acrescentam que com o avanço da idade, a cadência de passos tende a aumentar e a amplitude diminuir, ou seja, os idosos costumam diminuir o tamanho da passada, porém aumentam o número de passos realizados no período de um minuto. Nagasaki et al. ${ }^{25}$ corroboram essa ideia; contudo, também observaram que a cadência tendia a ser maior com o aumento da velocidade, conforme evidenciado neste estudo. Acredita-se que isso ocorra na tentativa de compensar a limitação de amplitude.

Ainda, para muitos idosos a autopercepção da manutenção da capacidade funcional e a intensidade do medo de sofrer quedas está intimamente ligada ao desempenho de uma velocidade de marcha eficaz ${ }^{26,27}$. Nessa perspectiva, em um estudo sobre quedas ${ }^{28}$, identificou-se uma relação significativa entre a prevalência de quedas e a redução dos níveis de mobilidade articular dos membros inferiores, pois se entende que as funções de equilíbrio e marcha se encontram intimamente relacionadas e precisam atuar de forma sincronizada para um desempenho satisfatório da deambulação.

Outra investigação similar ao estudo aqui apresentado, ao avaliar a marcha e equilíbrio de idosos institucionalizados, aponta que os déficits de equilíbrio e marcha são comuns nessa população, sendo mais frequentes no sexo feminino e nos idosos com idade mais avançada ${ }^{29}$.

Por intermédio da maior correlação observada neste estudo entre o desempenho na marcha e equilíbrio e a velocidade da marcha, sugere-se que em casos nos quais não seja possível aplicar o POMA, que avalia o desempenho na marcha e equilíbrio, ou que seja necessário aplicar um teste mais rápido e simples para avaliar a marcha, o teste da velocidade da marcha seria um bom parâmetro para identificar alterações, sendo sensível em detectar mudanças clínicas ${ }^{30}$.

\section{Conclusão}

Por meio deste estudo, em que se buscou correlacionar parâmetros de avaliação da marcha e equilíbrio em idosas institucionalizadas, observou-se que existe correlação entre os parâmetros analisados e, portanto, devem-se buscar estratégias para melhorar a cadência e velocida- 
de da marcha a fim de diminuir o risco de quedas entre idosas institucionalizadas.

Diante da maior correlação entre o desempenho na marcha e equilíbrio com a velocidade da marcha, em detrimento da cadência, sugerese que, na dificuldade clínica de aplicação do POMA, que avalia o desempenho na marcha e equilíbrio, pode-se aplicar, inicialmente, apenas o teste de velocidade da marcha, a fim de detectar alterações na mobilidade dos idosos.

Ao concluir este estudo, recomendam-se novas investigações que venham contemplar relações entre componentes da aptidão muscular e os parâmetros de avaliação da marcha e equilíbrio. Assim, será possível respaldar um melhor planejamento das intervenções com objetivo de manter a capacidade funcional dos idosos.

\section{Referências}

1. Camarano AA, Kanso S. As instituições de longa permanência para idosos no Brasil. Rev Bras Estud Popul. 2010; 27(1):233-52.

2. Yamamoto A, Diogo MJD. Os idosos e as instituições asilares do município de Campinas. Rev Latinoam Enferm. 2002;10(5):660-6.

3. Santos MLC, Andrade MC. Incidência de quedas relacionada aos fatores de riscos em idosos institucionalizados. Rev Baiana Saúde Pública. 2005;29(1):57- 8 .

4. Moreland JD, Richardson JA, Goldsmith $\mathrm{CH}$, Clase CM. Muscle weakness and falls in older adults: a systematic review and meta-analysis - California. Journal of American Geriatric Society, GSA. 2004;52(7):1121-9.

5. Perracini MR, Ramos LR. Fatores associados a quedas em uma coorte de idosos residentes na comunidade. Rev Saúde Pública. 2002;36(6):Edição especial.

6. Patla AE, Shumway-Cook A. Dimensions of mobility: defining the complexity and difficulty associated with community mobility- Scholary Review - J Aging Phys Act. 1999;82(7):670-81.

7. Perry J. Análise de marcha: marcha normal. São Paulo: Manole; 2005. p. 8-43.
8. Morais Filho MC, Reis RA, Kawamura CM. Avaliação do padrão de movimento dos joelhos e tornozelos durante a maturação da marcha normal. Acta Ortop Bras. [periódico na internet]. 2010[acesso em 2012 mar 1];18(1):23-5. Disponível em: http:// www.scielo.br/scielo.php?script=sci_arttext\&pid $=$ S1413-78522010000100004

9. Friedman PJ, Richmond DE, Baskett JJ. A prospective trial of serial gait speed as a measure of rehabilitation in the elderly. Age Ageing. 1988;17(1):227-35.

10. Farinatti PTV, Lopes LNC. Amplitude e cadência do passo e componentes da aptidão muscular em idosos: um estudo correlacional multivariado. Rev Bras Med Esporte . 2004 set/out;10(5).

11. Matsudo SM. Atividade física na promoção da saúde e qualidade de vida no envelhecimento. Rev Bras Educ Fís Esp. 2006;20(5):135-7.

12. Ribas DIR, Preis C, Dias AM, Chesani FH, Dias LA, Israel VL. Avaliação fisioterapêutica da marcha em idosas institucionalizadas. RUBS. 2005;1(4):33-7.

13. Born T, Boechat NS. A qualidade dos cuidados ao idoso institucionalizado. In: Freitas E V, Neri AL, editors. Tratado de geriatria e gerontologia. Rio de Janeiro: Vozes; 2002. p. 1131-41.

14. Bertolucci PHF, Brucki SMD, Campacci S, Juliano Y. O Mini-Exame do Estado Mental em uma população geral. Impacto da escolaridade. Arq Neuropsiquiatr. 1994;52:1-7.

15. Viel, E. O diagnóstico cinesioterapêutico: concepção, realização e transcrição na prática clínica e hospitalar. Barueri: Manole; 1998.

16. Abreu SSE, Caldas CP. Velocidade da marcha, equilíbrio e idade: um estudo cor relacional entre idosas praticantes e idosas não praticantes de um programa de exercícios terapêuticos. Rev Bras Fisioter. 2008;12(4): 324-30.

17. Tinetti ME. Performance-oriented assessment of mobility problems in elderly patients. J Am Geriatr Soc. 1986;34(2):114-26.

18. Gomes GC. Tradução, adaptação transcultural e exame das propriedades de medida da escala "Performanced-Oriented Mobility Assessment" (POMA) para uma amostra de idosos brasileiros institucionalizados (dissertação de Mestrado). Campinas: Unicamp; 2003. 
19. Brasil, Resolução n ${ }^{\circ} 196$, de 10 de outubro de 1996. Dispõe sobre as diretrizes e normas regulamentadoras de pesquisa envolvendo seres humanos. Conselho Nacional de Saúde. Brasília, DF 10 out. 1996.

20. Montero-Odasso M, Schapira M, Soriano ER, Varela M, Kaplan R, Camera LA, et al. Gait velocity as a single predictor of adverse events in healthy seniors aged 75 year and older. J Gerontology A Biol Sci Med Sci. 2005;60(10):1304-9.

21. Teixeira CS, Link DM, Ribeiro JK, Costa VP, Mota CB. Aspectos biomecânicos do caminhar em idosos. In: XVII Jornada Acadêmica Integrada. Anais Acadêmicos da Universidade Federal de Santa Maria (UFSM): Santa Maria; 2002.

22. Pijnappels M, Bobbert MF, van Dieën JH. Changes in walking pattern caused by the possibility of a tripping reaction. Gait Posture. 2001;14(1):11-8.

23. Soares AV, Matos FM, Laus LM, Susuki S. Estudo comparativo sobre a propensão de quedas em idosos institucionalizados e não-institucionalizados através do nível de mobilidade funcional. Rev Bras Fisioter. 2003;4(1):12-5.

24. Schenatto P, Milano D, Berlezi EM, Bonamigo ECB. Relação entre aptidão muscular e amplitude articular, por faixa etária, por faixa etária, na marcha do idoso. Rev Bras Geriatr Gerontol. 2009;12(3):377- 89 .
25. Nagasaki H, Itoh $\mathrm{H}$, Hashizume K, Furuna T, Maruyama H, Kinugasa T. Walking patterns and finger rhythm of older adults. Percept Mot Skills. 1996;82:435-47.

26. Maki BE. Gait changes in older adults: predictors of falls or indicators of fear. J Am Geriatr Soc. 1997;45:313-20.

27. Jylha M, Guralnik JM, Balfour J, Fried LP. Walking difficulty, walking speed, and age as predictors of self-rated health: the women's health and aging study. J Gerontol A Biol Sci Med Sci, 2001;56:M609-17.

28. Guimarães JMN, Farinatti PTV. Análise descritiva de variáveis teoricamente associadas ao risco de quedas em mulheres idosas. Rev Bras Med Esporte. 2005 set/ out;11(5):299-305.

29. Lojudice DC, Laprega MR, Gardezani PM, Vidal P. Equilíbrio e marcha de idosos residentes em instituições asilares do município de Catanduva, SP. Rev Bras Geriatr Gerontol. 2008;11(2):181-9.

30. Kollen B, Kwakkel G, Lindeman E. Time dependency of walking classification in stroke. Phys Ther. 2006;86(5):618-25 